\title{
Dual cascade and dissipation mechanisms in helical quantum turbulence
}

\author{
Patricio Clark di Leoni, ${ }^{1, *}$ Pablo D. Mininni, ${ }^{1, \dagger}$ and Marc E. Brachet ${ }^{2, \dagger}$ \\ ${ }^{1}$ Departamento de Física, Facultad de Ciencias Exactas y Naturales, Universidad de Buenos Aires and IFIBA, CONICET, \\ Ciudad Universitaria, 1428 Buenos Aires, Argentina \\ ${ }^{2}$ Laboratoire de Physique Statistique de l'Ecole Normale Supérieure associé au CNRS et aux Universités Paris 6 et 7, 24 Rue Lhomond, \\ 75237 Paris Cedex 05, France
}

(Received 15 November 2016; published 26 May 2017)

\begin{abstract}
While in classical turbulence helicity depletes nonlinearity and can alter the evolution of turbulent flows, in quantum turbulence its role is not fully understood. We present numerical simulations of the free decay of a helical quantum turbulent flow using the Gross-Pitaevskii equation at high spatial resolution. The evolution has remarkable similarities with classical flows, which go as far as displaying a dual transfer of incompressible kinetic energy and helicity to small scales. Spatiotemporal analysis indicates that both quantities are dissipated at small scales through nonlinear excitation of Kelvin waves and the subsequent emission of phonons. At the onset of the decay, the resulting turbulent flow displays polarized large scale structures and unpolarized patches of quiescence reminiscent of those observed in simulations of classical turbulence at very large Reynolds numbers.
\end{abstract}

DOI: 10.1103/PhysRevA.95.053636

\section{INTRODUCTION}

From the oceans to the solar wind, turbulence is widely found in nature. It is also observed in quantum fluids such as superfluids and Bose-Einstein condensates (BECs) [1]. Unlike classical flows, quantum flows have no viscosity and vorticity is concentrated along topological line defects with quantized circulation [2,3]. While similarities between these two types of turbulence exist (e.g., both display Kolmogorov spectrum $[4,5])$, there are also differences [6,7].

In classical turbulence helicity is an ideal invariant which measures how tangled vorticity field lines are [8]. It is known to deplete nonlinearities and energy transfer [9], slow down the onset of dissipation in decaying turbulence and affect its dissipation scale [10], play a role in convective storms [11], and display a dual direct cascade with the energy $[12,13]$. Its role in quantum turbulence is less clear. Efforts focus on determining if it is conserved by studying simple configurations of reconnecting vortex knots [14-18]. Numerical evidence indicates that in this case helicity is transferred from large to small scales [14,16], and that reconnection or the transfer of helicity can excite nonlinear interacting Kelvin waves $[17,19]$, which eventually may lead to a loss of helicity by sound emission. Research into the role of helicity in more complex quantum flows has been lacking, partly due to the difficulties of quantifying helicity in fully developed turbulent flows. However, developments in three-dimensional (3D) vortex tracking in helium experiments [20] and in knot generation in BECs [21] provide hopeful opportunities to tackle this problem.

We present massive numerical simulations of helical quantum turbulence using the Gross-Pitaevskii equation (GPE) as a model. A quantum version of the classical Arnold-BeltramiChildress (ABC) flow is introduced and used as initial condition to create a helical flow. We use different methods

\footnotetext{
*clark@df.uba.ar

†mininni@df.uba.ar

†brachet@lps.ens.fr
}

to quantify helicity, including the regularized helicity [17] which was shown to give results equivalent to the centerline helicity for simple knots, and to the classical helicity for flows with scale separation. We show that helicity is depleted as the incompressible kinetic energy. As in the classical case [12], both the incompressible energy and the helicity follow a Kolmogorov spectrum down to the intervortex distance. At smaller scales a bottleneck in the energy spectrum is followed by another Kolmogorov spectrum associated to a Kelvin waves cascade. Energy and helicity dissipation at coherent length scales is related to Kelvin waves damping by phonon emission. In physical space, the flow displays polarized large scale structures formed by a myriad of small scale knots, and unpolarized quiescent patches mimicking what is observed in isotropic and homogeneous classical flows at large Reynolds. These results open up questions about helicity in quantum flows. In particular, while successful theories for the energy spectrum exist [22], this is not yet the case for the helicity spectrum.

\section{GROSS-PITAEVSKII EQUATION}

The GPE describes the evolution of a zero-temperature condensate of weakly interacting bosons of mass $m$,

$$
i \hbar \partial_{t} \Psi=-\hbar^{2}(2 m)^{-1} \nabla^{2} \Psi+g|\Psi|^{2} \Psi,
$$

where $g$ is related to the scattering length. Madelung transformation $\Psi=\sqrt{\rho / m} \exp (\operatorname{im} \phi / \hbar)$ relates the wave function $\Psi$ to a condensate of density $\rho$ and velocity $\mathbf{v}=\nabla \phi$. Linearizing Eq. (1) around a constant $\Psi=\hat{\Psi}_{0}$ yields the Bogoliubov dispersion relation $\omega_{B}(k)=c k\left(1+\xi^{2} k^{2} / 2\right)^{1 / 2}$ for sound waves (or phonons) of speed $c=\left(g\left|\hat{\Psi}_{0}\right|^{2} / m\right)^{1 / 2}$, with dispersion taking place at lengths smaller than the coherence length $\xi=\left[g \hbar^{2}\left|\hat{\Psi}_{\mathbf{0}}\right|^{2} /(2 m)\right]^{1 / 2}$. The Onsager-Feynman quantum of velocity circulation around the $\Psi=0$ topological defect lines is $h / m$, and the vortex core size is of order $\xi$ [23].

The GPE conserves the total energy $E$, which can be decomposed as [24,25]

$$
E=E_{\text {kin }}+E_{\text {int }}+E_{\mathrm{q}},
$$


with the kinetic energy $E_{\text {kin }}=\left\langle|\sqrt{\rho} \mathbf{v}|^{2} / 2\right\rangle$, the internal energy $E_{\text {int }}=\left\langle c^{2}(\rho-1)^{2} / 2\right\rangle$, and the so-called quantum energy $E_{\mathrm{q}}=\left\langle c^{2} \xi^{2}|\nabla \sqrt{\rho}|^{2}\right\rangle$. The kinetic energy $E_{\text {kin }}$ can be also decomposed into compressible $E_{\text {kin }}^{\mathrm{c}}$ and incompressible $E_{\text {kin }}^{\mathrm{i}}$ components, using $(\sqrt{\rho} \mathbf{v})=(\sqrt{\rho} \mathbf{v})^{\mathrm{c}}+(\sqrt{\rho} \mathbf{v})^{\mathrm{i}}$ with $\nabla \cdot(\sqrt{\rho} \mathbf{v})^{\mathrm{i}}=0$.

\section{A. Helicity in quantum flows}

The definition of helicity in a classical flow is

$$
H=\int \mathbf{v} \cdot \omega d V
$$

where $\omega=\nabla \times \mathbf{v}$ is the vorticity. It follows from Madelung transformation that

$$
\omega(\mathbf{r})=\frac{h}{m} \int d s \frac{d \mathbf{r}_{0}}{d s} \delta\left(\mathbf{r}-\mathbf{r}_{0}(s)\right),
$$

where $\mathbf{r}_{0}(s)$ denotes the position of the vortex lines and $s$ the arclength. Thus vorticity in a quantum flow is a distribution concentrated along $\Psi=0$ topological line defects where $\mathbf{v}$ is ill behaved. In spite of this, some authors [15] compute $H$ by filtering fields to the largest scales or relying on the regularization introduced by the numerics. Other authors compute the "centerline helicity" by calculating the writhe and link, two topological quantities which quantify how knotted vortex lines are, but which require detailed extraction of all centerlines of the quantized vortices in the flow [14,16,26,27]. Some authors suggest to also add the twist of equal-phase surfaces (or else just the torsion) to this definition, but then the total helicity vanishes identically (or else smoothly formed inflection points change the helicity discontinuously) [18]. A method which yields the same results as the centerline helicity was introduced in [17] by using the fact that the velocity parallel to the quantized vortex has only an apparent singularity. The regular smooth velocity oriented along the vortex line is defined as $\mathbf{v}_{\text {reg }}=v_{\|} \mathbf{w} / \sqrt{w_{j} w_{j}}$, where

$$
v_{\|}=\frac{\hbar w_{j}\left[\left(\partial_{j} \partial_{l} \Psi\right) \partial_{l} \bar{\Psi}-\left(\partial_{j} \partial_{l} \bar{\Psi}\right) \partial_{l} \Psi\right]}{2 i m \sqrt{w_{l} w_{l}}\left(\partial_{m} \Psi\right)\left(\partial_{m} \bar{\Psi}\right)},
$$

and

$$
\mathbf{w}=\frac{\hbar}{i m} \nabla \bar{\Psi} \times \nabla \Psi
$$

(see the Appendix for a detailed derivation). The regularized helicity thus reads

$$
H_{r}=\int \mathbf{v}_{\mathrm{reg}} \cdot \boldsymbol{\omega} d V
$$

and is well defined in the sense of distributions [28], as the test function $\mathbf{v}_{\text {reg }}$ is smooth. This expression was proven useful even in flows with hundreds of thousands of knots.

\section{B. Numerical simulations}

The GPE is solved in its dimensionless form and all quantities presented here are dimensionless (see [25,29] for more details). All numerical simulations in this paper have mean density $\rho_{0}=1$. Physical constants in Eq. (1) are determined by $\xi$ and $c=2$, and the quantum of circulation $h / m=c \xi / \sqrt{2}$. Simulations were performed using $512^{3}, 1024^{3}$, and $2048^{3}$ grid points, in a domain of linear size $L=2 \pi$. The largest $2048^{3}$ GPE simulation has a healing length $\xi \approx 2.2 \times 10^{-3}$ and an intervortex distance $\ell \approx 8 \times 10^{-2}$ (computed as in [24,25]). As a comparison, in ${ }^{3} \mathrm{He}$ experiments the size of the vortex core is $\approx 10^{-8} \mathrm{~m}$, the intervortex distance is $\approx 10^{-5} \mathrm{~m}$, and the system size is of order $10^{-2} \mathrm{~m}$ [1]. Scale separation is smaller for BECs, which are also better modeled by the GPE. Although proper scale separation in a simulation is currently out of reach, the $2048^{3}$ run is a significant improvement over most simulations of quantum turbulence which have one order of magnitude difference between $L$ and $\xi$.

To compare the GPE simulations with classical ABC flows we also simulated the incompressible Navier-Stokes (NS) equation

$$
\partial_{t} \mathbf{u}+(\mathbf{u} \cdot \nabla) \mathbf{u}=-\nabla p+v \nabla^{2} \mathbf{u},
$$

with $\nabla \cdot \mathbf{u}=0$, using $512^{3}$ points and viscosity $v=6.5 \times$ $10^{-4}$. All equations were integrated using GHOST [30], a pseudospectral code with periodic boundary conditions, fourth-order Runge-Kutta to compute time derivatives, and the $2 / 3$ rule for dealiasing.

As initial condition we use a superposition of $k=1$ and $k=2$ basic $\mathrm{ABC}$ flows: $\mathbf{v}_{\mathrm{ABC}}=\mathbf{v}_{\mathrm{ABC}}^{(1)}+\mathbf{v}_{\mathrm{ABC}}^{(2)}$, with

$$
\begin{aligned}
\mathbf{v}_{\mathrm{ABC}}^{(k)}= & {[B \cos (k y)+C \sin (k z)] \hat{x}+[C \cos (k z)} \\
& +A \sin (k x)] \hat{y}+[A \cos (k x)+B \sin (k y)] \hat{z},
\end{aligned}
$$

and $(A, B, C)=(0.9,1,1.1) / \sqrt{3}$. The basic ABC flow is a $2 \pi$-periodic stationary solution of the Euler equation with maximal helicity. To build its quantum version we first take the flow with $B=C=0$ and use Madelung transformation to obtain a wave function $\Psi_{A, k}^{x, y, z}=\exp \{i[A \sin (k x) m / \hbar] y+$ $i[A \cos (k x) m / \hbar] z\}$, where $[a]$ stands for the nearest integer to $a$ to enforce periodicity. The wave function of the quantum ABC flow is then obtained as $\Psi_{\mathrm{ABC}}^{(k)}=\Psi_{A, k}^{x, y, z} \times \Psi_{B, k}^{y, z, x} \times$ $\Psi_{C, k}^{z, x, y}$. Finally, $\Psi_{\mathrm{ABC}}=\Psi_{\mathrm{ABC}}^{(1)} \times \Psi_{\mathrm{ABC}}^{(2)}$ corresponds to the initial flow $\mathbf{v}_{\mathrm{ABC}}$. In practice, to correctly set the initial density with defects along the vortex lines and to correct frustration errors arising from periodicity, following [24,25] we first evolve $\Psi_{\mathrm{ABC}}$ using the advected real GuinzburgLandau equation [31], whose stationary solutions are solutions of the GPE with minimal emission of acoustic energy.

\section{RESULTS}

In Fig. 1 we show the evolution of the incompressible kinetic energy $E_{k}^{i}$ and of the regularized helicity $H_{r}$ for the $1024^{3}$ and $2048^{3}$ GPE runs, and for the NS equation (with $\left.H_{r} \equiv H\right)$. In all cases, $E_{k}^{i}$ and $H_{r}$ remain approximately constant for the first turnover times while turbulence develops (the so-called "inviscid" phase in the decay of classical flows). The total vortex length $\ell_{v}$ peaks at the end of this phase, which ends slightly earlier for $H_{r}$ than for $E_{k}^{i}$. Afterwards, $H_{r}$ and $E_{k}^{i}$ decrease in what seems a self-similar decay, with different rates in each system. As total energy is conserved in GPE, the decay of $E_{k}^{i}$ is accompanied by a growth of the other components of the energy, in particular of $E_{k}^{c}$. Indeed, in quantum turbulence the decay of $E_{k}^{i}$ is expected to result from the emission of phonons [32], and thus from classical results [33] the decay in $E_{k}^{i}$ should also produce a decay in $H_{r}$. 


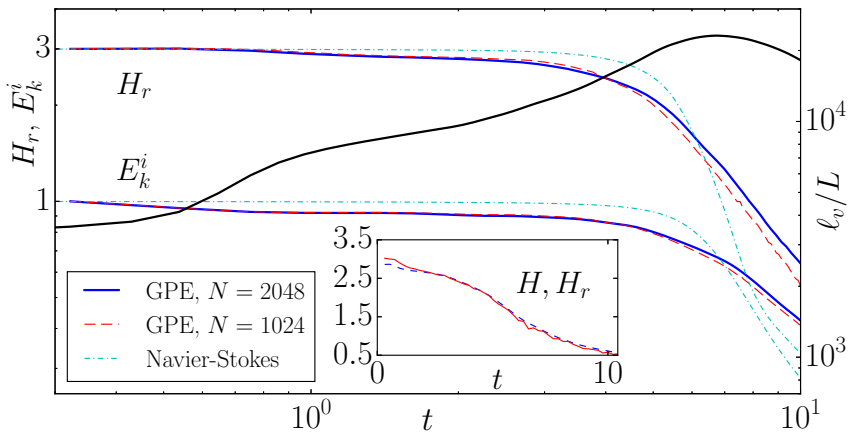

FIG. 1. Evolution of the incompressible energy $E_{k}^{i}$ and of the regularized helicity $H_{r}$ in the $1024^{3}$ and $2048^{3}$ GPE runs, and in Navier-Stokes. Note the early "inviscid" phase in which quantities are approximately constant. The solid black line shows the total vortex length in the $2048^{3}$ GPE run. Inset: $H_{r}(t)$ (dashed blue line) and the nonregularized helicity $H(t)$ (solid red line) in the $2048^{3}$ GPE run.

The inset in Fig. 1 compares $H$ (nonregularized) and $H_{r}$ in the $2048^{3}$ GPE run. Both are in good agreement, but $H_{r}$ is smoother and less noisy, making it a better fit to study the global evolution of helicity in quantum turbulence. However, the agreement between $H_{r}$ and $H$ allows us to use $H$ to compute spectra.

Figure 2 shows spectra of $E_{k}^{i}$ and $H$ at different times in the $2048^{3}$ GPE run. The spectra build up rapidly from the initial conditions, and the energy and helicity excite larger wave numbers as time increases. At $t=5$ both already display inertial ranges. At large scales they follow a power law compatible with a classical dual energy and helicity cascade [12], with Kolmogorov constant $C_{K} \approx 1$

$$
E(k) \approx \epsilon^{2 / 3} k^{-5 / 3}, \quad H(k) \approx \eta \epsilon^{-1 / 3} k^{-5 / 3},
$$
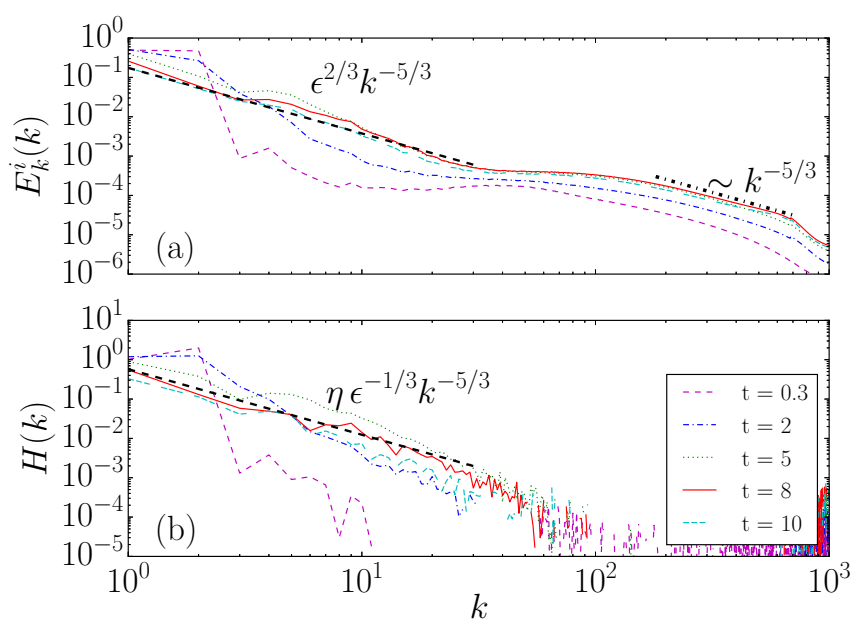

FIG. 2. Spectrum of the (a) incompressible kinetic energy, and (b) helicity in the $2048^{3}$ GPE run. At large scales both follow a scaling compatible with a classical dual cascade (thick dashed lines). At scales smaller than the intervortex scale $\left(k_{\ell} \approx 80\right)$ a second range compatible with a Kelvin wave cascade is observed in $E_{k}^{i}$ (thick dash-dotted line). The helicity spectrum broadens in time indicating a direct transfer.

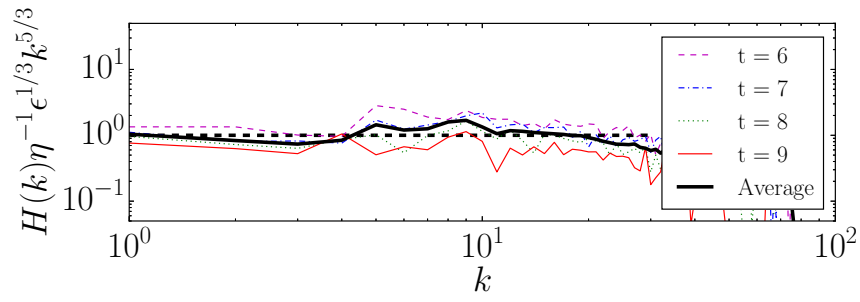

FIG. 3. Compensated helicity spectra in the $2048^{3}$ GPE run. The spectrum is compatible with $\eta \epsilon^{-1 / 3} k^{-5 / 3}$ scaling. The time-averaged spectrum is also shown.

and with $\epsilon$ and $\eta$ calculated directly using

$$
\epsilon=-d E_{k}^{i} / d t, \quad \eta=-d H / d t,
$$

from the data in Fig. 1 after the onset of decay. Around the mean intervortex scale $\left(k_{\ell} \approx 80\right) E_{k}^{i}$ diplays a bottleneck compatible with predictions in [22]. This bottleneck is followed by an inertial range $\sim k^{-5 / 3}$ predicted for a Kelvin wave cascade $[22,34]$ and which below is confirmed for the lower resolution run. Figure 3 shows compensated helicity spectra, which corroborates the behavior observed in Fig. 2.

Of particular interest is the evolution of $H(k)$. For early times $H$ is concentrated at low wave numbers, as expected for the initial condition. But later it is transferred to larger wave numbers as the cascadelike spectrum develops. While there is no rigourous proof of conservation of helicity in quantum flows, note that using the Hasimoto transformation [35] the evolution of a vortex line can be mapped into a nonlinear Schrödinger equation which conserves momentum. But momentum of a vortex line (e.g., the translation of the centerline in the direction of vorticity) can result in net helicity. Thus, vortex lines evolution could indeed conserve helicity (except for depletion by emission of phonons). At small scales $H(k)$ displays wild fluctuations (in amplitude and sign), which is to be expected as the nonregularized helicity is ill behaved at those scales. The fact that the helicity dynamics, at least at large scales, of a quantum flow mimics those of a classical one is remarkable. But it also begs the question of what happens to the helicity at scales smaller than the intervortex distance. Indications exist that Kelvin waves carry helicity [14,17], but such a possibility requires confirmation of their presence.

To verify this, as well as phonons being the dissipation mechanism for $E_{k}^{i}$ and $H$, we must detect Kelvin and sound waves. To do this we use the spatiotemporal spectrum [29], i.e., the four-dimensional power spectrum of a field as a function of wave vector and frequency. The spectrum allows quantification of how much power is in each mode $(\mathbf{k}, \omega)$, and waves can be separated from the rest as they satisfy a known dispersion relation $\omega(\mathbf{k})$. As its computation requires huge amounts of data (i.e., storage of fields resolved in space and time), we compute it for the $512^{3} \mathrm{GPE}$ run. Figure 4 shows this spectrum (after integration in $\mathbf{k}$ using isotropy to obtain dependency on $k$ and $\omega$ ) for $\rho$, and zooms for small $k$ for $E_{k}^{i}$ and $E_{k}^{c}$, for early and late times (respectively, $t \in[0,2]$ and $t \in[8,10]$ ). The dispersion relation of Kelvin $\omega_{K}(k)$ [36] and sound waves $\omega_{B}(k)$ are shown. Note that, compared with the $2048^{3}$ run, $\xi$ in this run is four times larger, and values of $k$ are four times smaller. 

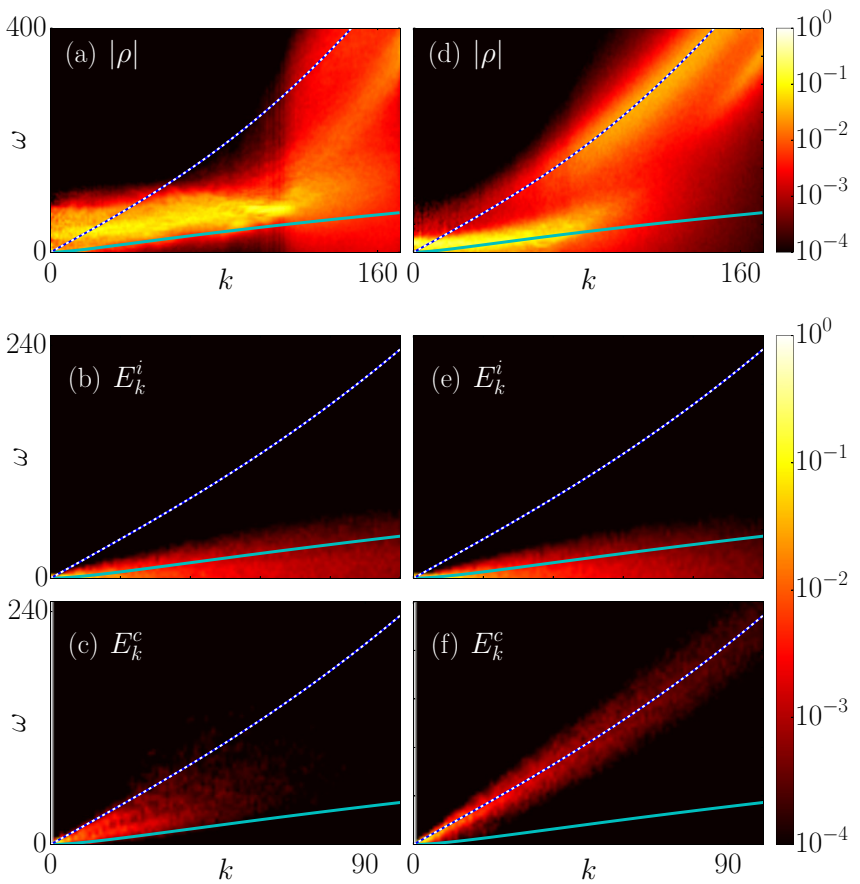

FIG. 4. Spatiotemporal power spectra for the $512^{3}$ GPE run between $t=0$ and 2 for (a) the mass density $\rho$, and zooms between $k=0$ and 100 for (b) the incompressible and (c) compressible velocity. Same for late times $(t \in[8,10])$ are shown in (d), (e), and (f). The solid (green) curve is the dispersion relation of Kelvin waves, while the dotted (white) curve corresponds to sound waves.

At early times, power in the spatiotemporal spectrum of $\rho$ is broadly spread over modes that do not correspond to waves. $E_{k}^{i}$ shows some excitations compatible with Kelvin waves, and $E_{k}^{c}$ has little energy with no phonon excitation. At late times power in fluctuations of $\rho$ moves towards the Kelvin wave dispersion relation up to $k \approx 80$, and then the power jumps towards the dispersion relation of phonons. The spectra $E_{k}^{i}$ and $E_{k}^{c}$ confirm this picture, with power concentrating in $E_{k}^{c}$ in the vicinity of the sound dispersion relation. Exploration of these spectra for different time ranges shows that as time evolves more energy goes from Kelvin wave modes to phonons. While this analysis is performed at lower resolution and thus wave numbers for the transition are smaller than in the $2048^{3}$ run, the spectra confirm the dynamics in Figs. 1 and 4: with time, energy and helicity go from large to smaller scales in which Kelvin waves are excited, and they are finally dissipated into phonons.

This can be further confirmed by visualizing vortices in real space at the onset of decay. Figure 5 shows a three-dimensional rendering of quantum vortices at $t \approx 2.5$ in the $2048^{3} \mathrm{GPE}$ run. Large-scale eddies, formed up by a myriad of small-scale and knotted vortices, emerge. Similar behavior has been observed at finite temperature quantum turbulence simulations, where the bundle was correlated with high vorticity in the normalfluid component [37,38]. At zero temperature two results [39-41] also hinted at this behavior, but in none was the fine structure of the vortex bundle resolved. More importantly, the large scale flow shows inhomogeneous regions with high density of vortices and quiet regions with low density. These large-scale patches were not present in the initial conditions

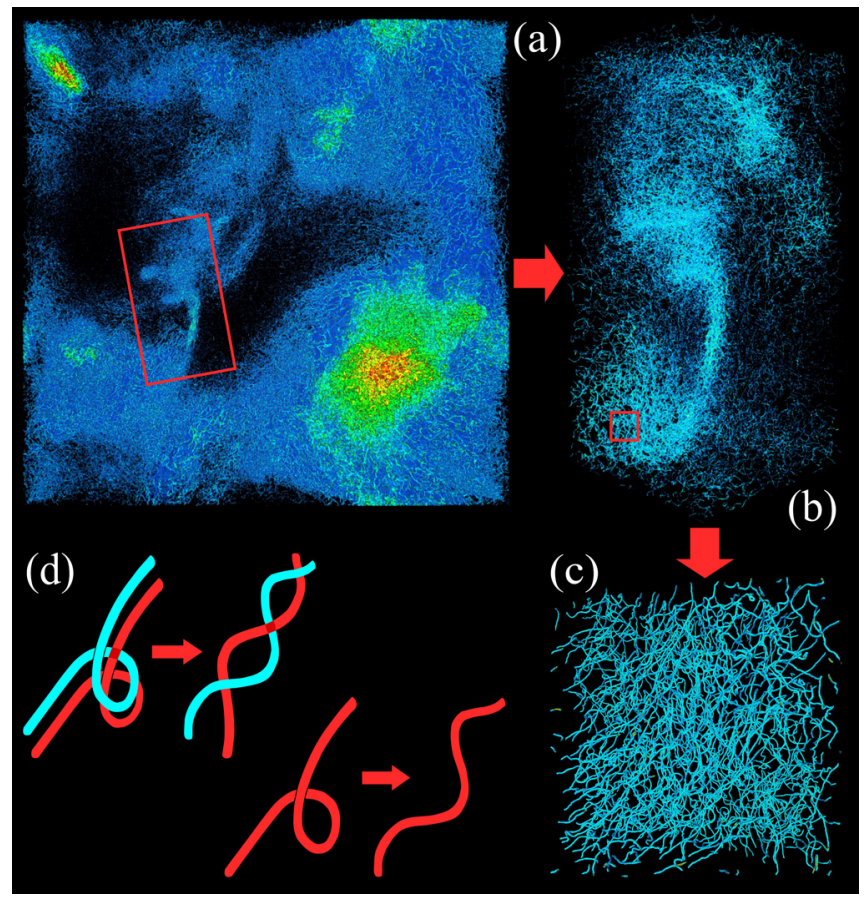

FIG. 5. Three-dimensional rendering of vortex lines at the onset of the decay in the $2048^{4}$ GPE run of (a) a slice of the full box, and successive zooms in (b) and (c) into the regions indicated by the (red) rectangles. (d) Sketch of the transfer of helicity from writhe to twist in a bundle of vortices, and for an individual vortex.

(which have homogeneously distributed vortices) and are created by the evolution as shown below. The patches are reminiscent of those observed in isotropic and homogeneous turbulence at high resolution in nonhelical [42] and helical [43] flows, further confirming the similarity between quantum and classical turbulence at scales larger than the intervortex separation.

The spontaneous emergence of large-scale correlations in the system can be confirmed by the spatial correlation

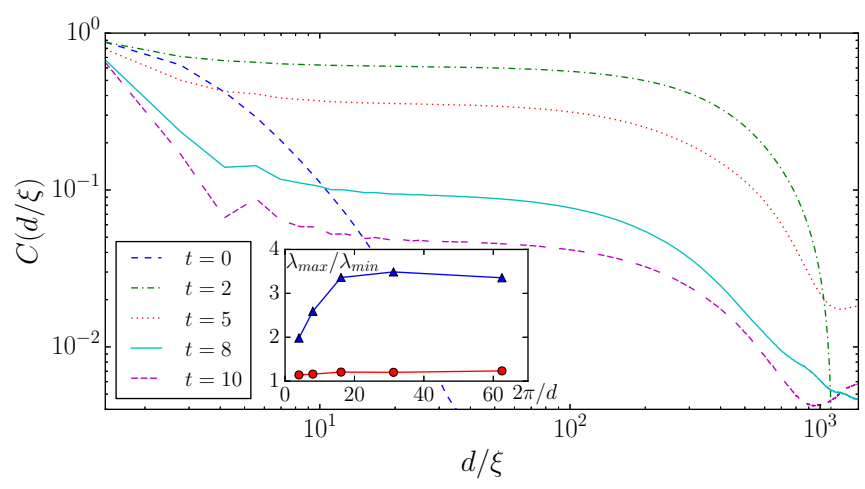

FIG. 6. Correlation function of $\rho$ in the $2048^{3}$ GPE run. At $t=0$ it decays rapidly in units of the healing length $\xi$, but then quickly develops long-range correlations. Inset: Ratio of eigenvalues $\lambda_{\max } / \lambda_{\min }$ as a function of $2 \pi / d$, with $d$ the size of the box used for the average (blue triangles: regions with structures; red triangles: regions of quiescence). 
function

$$
C(d)=\left\langle\left[\rho(\mathbf{x}+d \hat{x})-\rho_{0}\right]\left[\rho(\mathbf{x})-\rho_{0}\right]\right\rangle,
$$

shown in Fig. 6. This correlation function is related to the internal energy spectrum by the Wiener-Khinchin theorem. At $t=0, C(d)$ decays rapidly in units of the healing length $\xi$, and it is dominated by the vortex core size. But the system rapidly develops long-range correlations (up to $\approx 1000 \xi$ ) and later $C$ decays in a self-similar way. Furthermore, computing the ratio of eigenvalues $\tau=\lambda_{\max } / \lambda_{\min }$ for the tensor $\left\langle\partial_{i} \rho \partial_{j} \rho\right\rangle$ averaged in boxes of size $1 / 10$ of the linear domain size typically yields $\tau \approx 3$ in regions with large scale structures and $\tau \approx 1$ in quiescent regions, indicating anisotropy and a copious vortex polarization in the former (see Fig. 6 inset).

\section{CONCLUSIONS}

The results indicate that helicity can be conserved in quantum turbulence at large scales and as it is transferred towards smaller scales (see Fig. 2), but eventually it decays through the emission of phonons produced by a Kelvin wave cascade (Fig. 4). We can draw a comparison with the classical case, where now a bundle of quantum vortices (as seen in Fig. 5) would play the role of classical vortex tubes. Tubes, in contrast to lines, add an extra degree of freedom to the helicity: their twist. Thus in the classical and quantum cases, large scale helicity can be transformed from writhe to twist for a bundle of vortices [see Fig. 5(d)]. But for individual quantum vortices, the transfer (e.g., through reconnection) would result in the excitation of a Kelvin wave which can eventually be damped. This indicates that individual quantum vortex lines behave like classical vortex tubes with a mechanism to relax the twist, and as such, the correct analogy between classical and quantum flows only holds for scales larger than $\ell$ for which bundles of quantum vortices behave as classical vortex tubes.

\section{ACKNOWLEDGMENTS}

The authors acknowledge financial support from Grant No. ECOS-Sud A13E01 and from computing hours in the CURIE supercomputer granted by Project TGCC-GENCI No. x20152a7493.

\section{APPENDIX: DERIVATION OF THE REGULARIZED VELOCITY}

To calculate the helicity in a quantum flow we need information of both the velocity and the vorticity along the vortex lines. This is problematic as both quantities have singularities along those lines. Therefore, we need to regularize one of them in order to have a well-behaved integral for the helicity (in the sense of distributions [28]). Although in principle it may seem possible to regularize any of the two fields, the choice of regularizing the velocity and not the vorticity is not arbitrary. In the Gross-Pitaevskii equation, the vorticity is correctly described by a distribution. Instead, the only component of the velocity that is not well behaved is the one perpendicular to the vortex line. But for the calculation of the helicity we need the parallel component, whose problem is to have a $0 / 0$ indeterminacy in its definition.
Thus, regularizing the velocity allows us to keep its well defined component which contributes to the helicity, while leaving the vorticity as a Dirac $\delta$ distribution also allows us to not bother with the values of the regularized field outside the vortex line, which should give no contribution to the helicity. Here we outline a detailed explanation of how to derive the regularized velocity, from which the expression of the regularized helicity follows immediately.

The velocity of the superfluid is given by

$$
\mathbf{v}=\frac{\hbar}{2 i m} \frac{\bar{\Psi} \nabla \Psi-\Psi \nabla \bar{\Psi}}{\Psi \bar{\Psi}} .
$$

Without loss of generality we can suppose that there is a vortex line going through $\mathbf{r}=0$ (the radial cylindrical vector) in the direction of the $z$ axis. Let us define the unit vector basis $\left(\hat{\mathbf{e}}_{x}, \hat{\mathbf{e}}_{y}, \hat{\mathbf{e}}_{z}\right)$. The existence of a vortex line passing through $\mathbf{r}=0$ and pointing in the $z$ direction implies that $\Psi(0)=0$, $\bar{\Psi}(0)=0, \hat{\mathbf{e}}_{z} \cdot \nabla \Psi(0)=0$, and $\hat{\mathbf{e}}_{z} \cdot \nabla \bar{\Psi}(0)=0$. Thus $\nabla \Psi(0)$ and $\nabla \bar{\Psi}(0)$ are linear combinations of $\hat{\mathbf{e}}_{x}$ and $\hat{\mathbf{e}}_{y}$. Taylor expanding to first order the numerator and denominator of the above expression for $\mathbf{v}(\mathbf{r})$ around $\mathbf{r}=0$ one finds

$$
\begin{gathered}
\Psi(x, y, z)=x \partial_{x} \Psi(0)+y \partial_{y} \Psi(0)+O\left(\mathbf{r}^{2}\right), \\
\bar{\Psi}(x, y, z)=x \partial_{x} \bar{\Psi}(0)+y \partial_{y} \bar{\Psi}(0)+O\left(\mathbf{r}^{2}\right), \\
\nabla \Psi(x, y, z)=\nabla \Psi(0)+\mathbf{r} \cdot \nabla(\nabla \Psi)(0)+O\left(\mathbf{r}^{2}\right), \\
\nabla \bar{\Psi}(x, y, z)=\nabla \bar{\Psi}(0)+\mathbf{r} \cdot \nabla(\nabla \bar{\Psi})(0)+O\left(\mathbf{r}^{2}\right) .
\end{gathered}
$$

After replacing the above expressions in Eq. (A1) and dropping quadratic terms, the perpendicular $(x$ and $y)$ components of the velocity diverge in the limit $\mathbf{r} \rightarrow 0$, as $\mathbf{v}_{\perp}$ reads

$$
\mathbf{v}_{\perp}(\mathbf{r})=\frac{\hbar}{2 i m}\left(\frac{\nabla \Psi(0)}{x \partial_{x} \Psi(0)+y \partial_{y} \Psi(0)}-\text { c.c. }\right) \text {. }
$$

On the other hand, the velocity component parallel to the centerline vorticity $v_{\|}(\mathbf{r})=\mathbf{v}(\mathbf{r}) \cdot \hat{\mathbf{e}}_{z}$ reads

$$
\begin{aligned}
& v_{\|}(\mathbf{r}) \\
& =\frac{\hbar}{2 i m}\left(\frac{x\left(\partial_{x z} \Psi\right)(0)+y\left(\partial_{y z} \Psi\right)(0)+z\left(\partial_{z z} \Psi\right)(0)}{x \partial_{x} \Psi(0)+y \partial_{y} \Psi(0)}-\text { c.c. }\right),
\end{aligned}
$$

which is finite in the limit $\mathbf{r} \rightarrow 0$. This last expression for $v_{\|}(\mathbf{r})$ can be seen as resulting from l'Hôpital's rule applied to the limit of $v_{\|}(\mathbf{r})$ when $\mathbf{r} \rightarrow 0$ in the direction $(x, y, z)$. The limit obviously depends on the direction as, in deriving the above formulas, the only hypotheses we have made are that $\Psi$ is sufficiently differentiable and has a zero line directed toward $z$.

In order to turn the above expression into a workable ansatz for $v_{\|}(0)$, we need to pick a reasonable direction along which $\Psi$ will have a significant variation. The simplest vectors we have at point $\mathbf{r}=0$, perpendicular to the vortex line and satisfying the condition, are $\nabla \Psi(0)$ and $\nabla \bar{\Psi}(0)$. Thus we can multiply the first term in the right-hand side of Eq. (A7) by $\nabla \bar{\Psi}$, and its complex conjugate by $\nabla \Psi$ in order to maintain the reality of the velocity field. In this way we arrive at the following 
expression:

$$
v_{\|}(0)=\frac{\hbar}{2 m i}\left(\frac{\partial_{x} \bar{\Psi} \partial_{x z} \Psi+\partial_{y} \bar{\Psi} \partial_{y z} \Psi+\partial_{z} \bar{\Psi} \partial_{z z} \Psi}{\partial_{x} \bar{\Psi} \partial_{x} \Psi+\partial_{y} \bar{\Psi} \partial_{y} \Psi+\partial_{z} \bar{\Psi} \partial_{z} \Psi}-\text { c.c. }\right) \text {. }
$$

A first check that this ansatz is reasonable is to plug in $\Psi \sim(x+i y) e^{i z U_{z} m / \hbar}$ and explicitly verify that this gives $v_{\|}(0)=U_{z}$. Further validations were performed in [17], where it was shown that the helicity computed with the regularized velocity agrees with the topological definitions of writhe, link, and twist. Also, in [17] it was shown that this expression gives the correct value of helicity for different knots, and that in quantum flows with helicity it gives a value that matches the helicity in the equivalent classical large-scale helical flow.

As a final remark, it is important to note that for arbitrarily aligned vortex lines, the direction parallel to the vortex line $(\hat{z}$ in the particular case considered above) can be easily obtained by doing the vector product between $\nabla \Psi$ and $\nabla \bar{\Psi}$.
[1] C. F. Barenghi, L. Skrbek, and K. R. Sreenivasan, Proc. Natl. Acad. Sci. USA 111, 4647 (2014).

[2] R. P. Feynman, in Progress in Low Temperature Physics, edited by C. J. Gorter (Elsevier, New York, 1955), Vol. 1, pp. 17-53.

[3] R. J. Donnelly, Quantized Vortices in Helium II (Cambridge University Press, Cambridge, England, 1991).

[4] J. Maurer and P. Tabeling, Europhys. Lett. 43, 29 (1998).

[5] J. Salort, B. Chabaud, E. Lévêque, and P.-E. Roche, Europhys. Lett. 97, 34006 (2012).

[6] M. S. Paoletti, M. E. Fisher, K. R. Sreenivasan, and D. P. Lathrop, Phys. Rev. Lett. 101, 154501 (2008).

[7] A. C. White, C. F. Barenghi, N. P. Proukakis, A. J. Youd, and D. H. Wacks, Phys. Rev. Lett. 104, 075301 (2010).

[8] H. K. Moffatt, J. Fluid Mech. 35, 117 (1969).

[9] R. H. Kraichnan, J. Fluid Mech. 59, 745 (1973).

[10] J. C. André and M. Lesieur, J. Fluid Mech. 81, 187 (1977).

[11] D. K. Lilly, J. Atmos. Sci. 43, 126 (1986).

[12] A. Brissaud, U. Frisch, J. Leorat, M. Lesieur, and A. Mazure, Phys. Fluids 16, 1366 (1973).

[13] H. K. Moffatt and A. Tsinober, Annu. Rev. Fluid Mech. 24, 281 (1992).

[14] M. W. Scheeler, D. Kleckner, D. Proment, G. L. Kindlmann, and W. T. M. Irvine, Proc. Natl. Acad. Sci. USA 111, 15350 (2014).

[15] S. Zuccher and R. L. Ricca, Phys. Rev. E 92, 061001 (2015).

[16] D. Kleckner, L. H. Kauffman, and W. T. M. Irvine, Nat. Phys. 12, 650 (2016).

[17] P. Clark di Leoni, P. D. Mininni, and M. E. Brachet, Phys. Rev. A 94, 043605 (2016).

[18] R. Hnninen, N. Hietala, and H. Salman, Sci. Rep. 6, 37571 (2016).

[19] E. Fonda, D. P. Meichle, N. T. Ouellette, S. Hormoz, and D. P. Lathrop, Proc. Natl. Acad. Sci. USA 111, 4707 (2014).

[20] D. P. Lathrop (private communications).

[21] D. S. Hall, M. W. Ray, K. Tiurev, E. Ruokokoski, A. H. Gheorghe, and M. Möttönen, Nat. Phys. 12, 478 (2016).

[22] V. S. L'vov and S. Nazarenko, J. Exp. Theor. Phys. Lett. 91, 428 (2010).
[23] N. P. Proukakis and B. Jackson, J. Phys. B 41, 203002 (2008).

[24] C. Nore, M. Abid, and M. E. Brachet, Phys. Fluids 9, 2644 (1997).

[25] C. Nore, M. Abid, and M. E. Brachet, Phys. Rev. Lett. 78, 3896 (1997).

[26] C. E. Laing, R. L. Ricca, and D. W. L. Sumners, Sci. Rep. 5, 9224 (2015).

[27] A. Villois, D. Proment, and G. Krstulovic, Phys. Rev. E 93, 061103 (2016).

[28] M. J. Lighthill, An Introduction to Fourier Analysis and Generalised Functions (Cambridge University Press, Cambridge, England, 1958).

[29] P. Clark di Leoni, P. D. Mininni, and M. E. Brachet, Phys. Rev. A 92, 063632 (2015).

[30] P. D. Mininni, D. Rosenberg, R. Reddy, and A. Pouquet, Parallel Comput. 37, 316 (2011).

[31] $\partial_{t} \Psi=\frac{\hbar}{2 m} \nabla^{2} \Psi+\left(\frac{g \rho_{0}}{m}-g|\Psi|^{2}-\frac{m \mathbf{v}_{\mathrm{ABC}}^{2}}{2 \hbar}\right) \Psi-i \mathbf{v}_{\mathrm{ABC}} \cdot \nabla \Psi$.

[32] W. F. Vinen and J. J. Niemela, J. Low Temp. Phys. 128, 167 (2002).

[33] T. Teitelbaum and P. D. Mininni, Phys. Fluids 23, 065105 (2011).

[34] G. Krstulovic, Phys. Rev. E 86, 055301 (2012).

[35] H. Hasimoto, J. Fluid Mech. 51, 477 (1972).

[36] $\omega_{K}(k)=\left\{1 \pm\left[1+k a K_{0}(k a) / K_{1}(k a)\right]^{1 / 2}\right\} \sqrt{2} c \xi / a^{2}$, where $a$ is the vortex core radius and $K_{0}, K_{1}$ are modified Bessel functions. This dispersion relation is quadratic for small $k$ and linear for large $k$.

[37] K. Morris, J. Koplik, and D. W. I. Rouson, Phys. Rev. Lett. 101, 015301 (2008).

[38] S. K. Nemirovskii, Phys. Rep. 524, 85 (2013).

[39] N. Sasa, T. Kano, M. Machida, V. S. L'vov, O. Rudenko, and M. Tsubota, Phys. Rev. B 84, 054525 (2011).

[40] A. W. Baggaley, J. Laurie, and C. F. Barenghi, Phys. Rev. Lett. 109, 205304 (2012).

[41] A. W. Baggaley, C. F. Barenghi, A. Shukurov, and Y. A. Sergeev, Europhys. Lett. 98, 26002 (2012).

[42] T. Ishihara, T. Gotoh, and Y. Kaneda, Annu. Rev. Fluid Mech. 41, 165 (2009).

[43] P. D. Mininni, A. Alexakis, and A. Pouquet, Phys. Rev. E 74, 016303 (2006). 